Ann. Zootech., I972, 21 (3), 40I-4I4.

\title{
INFLUENCE DU POIDS D'ABATTAGE ET DU SEXE SUR LES PERFORMANCES DE BOUCHERIE DES AGNEAUX ISSUS DU CROISEMENT MANCHEGO $\times$ RASA ARAGONESA
}

\author{
F. COLOMER-ROCHER et M. ESPEJO-DIAZ \\ Departamento de Producción Animal, \\ Centro de Investigación y Desarrollo Agrario del Ebro, \\ Apartado 202, \\ Zaragoza - Espagne
}

RÉSUMÉ

Soixante agneaux issus d'un croisement Manchego $\times$ Rasa Aragonesa ont été répartis en six lots de ro animaux : 3 lots par sexe (mâles et femelles) constitués en fonction du poids à l'abattage $(22 \mathrm{~kg}, 26 \mathrm{~kg}$ et $30 \mathrm{~kg}$ ). Tous les animaux ont été élevés dans des conditions identiques. On a étudié la croissance des agneaux et le rendement, l'état d'engraissement, la conformation et l'importance des morceaux de découpe des carcasses.

Du fait de la meilleure précocité des femelles pour le type de croisement étudié, dans les conditions d'élevage envisagées, et en vue de la production de carcasses lourdes, leur poids optimum d'abattage se situe à $26 \mathrm{~kg}$, tandis qu'on peut atteindre des poids supérieurs à $30 \mathrm{~kg}$ pour les mâles.

\section{I. - INTRODUCTION}

Sur le marché de la viande ovine, on constate une évolution générale de la demande vers des carcasses lourdes et bien conformées. Ainsi en France, le poids optimum se situe entre I $_{5}$ et I $8 \mathrm{~kg}$ (FLAMANT et BOCCARD, I966) et au Royaume-Uni entre 15 et $24 \mathrm{~kg}$ (LuCAS, I97I). Le marché des U. S. A. exige, quant à lui, des carcasses d'un poids supérieur à $25 \mathrm{~kg}$ (LAmbuTh, KEMP et GLIMP, r970). Par contre, dans les régions méditerranéennes et dans le cas précis de 1'Espagne, la demande a orienté jusqu'à présent la production vers des carcasses légères, selon la croyance, à notre avis injustifiée, que la qualité de la carcasse est fonction, exclusivement, de son poids. Les conséquences de cette situation, sur le volume de la production de viande ovine espagnole et la situation đu marché, ont été discutées par SANCHEZBELDA (I970). 
Pour raisonner un programme de production de carcasses lourdes, permettant l'augmentation de la production de viande d'agneau sans variation de l'effectif du cheptel ovin, de nombreux travaux portant sur la croissance et le développement des agneaux et leurs effets sur les caractéristiques des carcasses sont intéressants à considérer. Ainsi, Palsson et VerGES (I952) ont étudié les effets du niveau d'alimentation, Boccard et Duplan (I96x) de la vitesse de croissance, Desvignes, CatTinVidal et Poly (ig66) et Flamant, Cattin-Vidal et Poly (ig67) de la race, Clarke et Mc MEEKAN (I952) du sexe, et KEMP et al. (I970) de la castration.

Dans le cadre d'un travail sur la production d'agneaux de boucherie par des brebis aragonaises (en race pure ou en croisement), nous étudions pour notre part l'effet du poids à l'abattage, en fonction du sexe, sur les qualités bouchères des carcasses (conformation et état d'engraissement).

\section{II. - MATÉRIFL E'T MÉTHODES}

L'étude porte sur 60 agneaux (30 mâles entiers et 30 femelles) nés simples et de brebis adultes Rasa Aragonesa (1) saillies par des bélicrs de race pure Manchega. Avec les agneaux de chaque sexe on a constitué au hasard, au cours du premier mois après la naissance, trois lots de io animaux destinés à être abattus respectivement à 22,26 et $30 \mathrm{~kg}$.

Les agneaux ont été élevés avec leur mère en stabulation permanente et ont été sevrés au poids de $22 \mathrm{~kg}$. Chaque lot (sexe $\times$ poids d'abattage) a été conduit et alimenté séparément, mais de façon identique, de l'agnelage à l'abattage cies agneaux. Pendant la période d'allaitement, on a distribué une ration journalière correspondant aux quantités movennes suivantes par brebis: paille $500 \mathrm{~g}$, foin de luzerne $800 \mathrm{~g}$, aliment concentré commercial $300 \mathrm{~g}$, orge $200 \mathrm{~g}$, et complément minéral Io $\mathrm{g}$ (soit $0,95 \mathrm{UF}$ et $\mathrm{I} 32 \mathrm{~g}$ MAD). En outre, les agneaux ont reçu, en moyenne, $200 \mathrm{~g}$ de foin de luzerne par tête et ont eu accès, à volonté, avant et après le sevrage, à un aliment concentré commercial distribué en auge ( 17 p. Ioo de protéine brute) : les quantités consommées ont été mesurées globalement pour chaque lot.

Les agneaux furent pesés à la naissance, puis chaque semaine, jusqu'à la fin de l'expérience. Les abattages sont intervenus dans l'après-midi du jour de la pesée hebdomadaire pour les animaux atteignant le poids correspondant à leur lot, et dans un abattoir situé à $+\mathrm{km}$ de la bergerie. La durée du jeûne a été de huit heures.

Les carcasses, comprenant les reins et la graisse périrénale, furent pesées à chaud et à froid, 24 heures après réfrigération en chambre froide à $-4^{\circ} \mathrm{C}$. Sur la carcasse froide suspendue par les jarrets et en maintenant un écartement constant de $12,5 \mathrm{~cm}$ entre les métatarses, on a effectué les mesures F, G, Wr, K et Th (proposées par PALssox, 1939 et décrites à nouveau par BoccARD, Dumont et PEYron, 1964) qui permettent d'avoir une appréciation objective de la conformation de la carcasse.

Au moment de l'abattage, le sang, la peau et tous les organes du cinquième quartier furent pesés après le vidage du contenu du tube digestif. Lc poids vif vide a été estimé par addition, au poids de la carcasse chaude, du poids des organes, de la peau et du sang.

L'état d'engraissement a été estimé par le poids froid de la graisse mésentérique, périrénale et pelvienne et par l'épaisseur de la graisse de couverture dans le prolongement de l'axe le plus court du longissimus dorsi au niveau de la première vertèbre lombaire (mesure C) et au niveau de la première vertèbre sacrée (mesure $\mathrm{C}^{\prime}$ ).

Les moitiés gauche des carcasses obtenues par section longitudinale ont été découpées d'après la découpe traditionnelle d'Aragon, schématisée figure I. Afin de faciliter la compréhension du texte, nous avons donné la traduction française des termes espagnols, bien que les bases anatomiques des morceaux des découpes aragonaise et française ne soient pas toujours identiques.

(1) La race Rasa Aragonesa est une race rustique de petit format dont le poids des brebis adultes varie de 30 à $45 \mathrm{~kg}$ et celui des béliers de 45 à $70 \mathrm{~kg}$. La race Manchega est plus lourde (40 à $70 \mathrm{~kg}$ pour les brebis et 60 à $\mathrm{I}$ Io $\mathrm{kg}$ pour les béliers). 
Le rendement commercial a été calculé par le rapport du poids de la carcasse froide au poids vif en bergerie et le rendement vrai par le rapport du poids de la carcasse chaude au poids vif vide estimé.
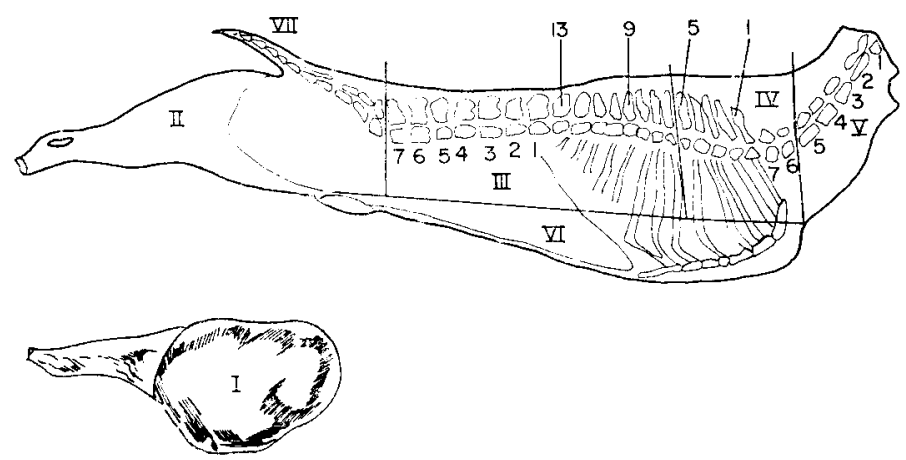

Fig. x. - Découpe de la carcasse en Aragon
I. Espalda (Épaule)
II. Pierna (Gigot)
III. Costillar (Côtes + Filet)
IV. Badal (Carré découvert)
V. Cuello (Collier)
VI. Bajos (Poitrine)
VII. Cola (Queue)

Chacun des critères mesurés a été analysé selon une analyse de variance équilibrée où l'on tient compte du sexe (mâle ou femelle), du traitement (lots d'abattage à 22, 26 ou $30 \mathrm{~kg}$ ) et de leur interaction.

\section{III. - RÉSUITATS E'T DISCUSSION}

Pour chacun des critères étudiés, on n'observe pas d'interaction significative entre le poids à l'abattage et le sexe, sauf pour l'âge à l'abattage. En conséquence, les cellules " sexe - poids à l'abattage " comprenant un nombre égal d'animaux, nous discutons les résultats concernant l'influence du sexe indépendamment du poids à l'abattage, et ceux relatifs à l'influence du poids à l'abattage sans tenir compte du sexe.

\section{I. - Croissance et consommation d'aliments}

Les courbes de croissance des animaux abattus à $30 \mathrm{~kg}$ sont représentées dans la figure 2 où on observe que les agneaux mâles présentent une croissance constante jusqu'à ce poids. Celle des femelles, pratiquement égale à celle des mâles jusqu'à l'âge d'un mois, devient plus faible ensuite. Le ralentissement de la croissance des femelles est particulièrement sensible $(\mathrm{P}<0,05)$ à partir du poids vif de $22 \mathrm{~kg}$ et de 1'âge de 80 jours, ce qui semble être dû à la plus grande précocité des femelles. Corrélativement, le temps nécessaire pour atteindre le poids vif de $30 \mathrm{~kg}$ a été nettement plus long chez les femelles que chez les mâles (2I jours, différence hautement significative, tab1. I). Plusieurs auteurs (PALsson et VERGES, I952 ; DEsvignes, CatTinVidal et Poly, Ig66; Flaman', CaTTin-Vidal et Poly, I967) ont aussi constaté qu'une plus grande précocité physiologique, manifestée ici sur les femelles par un ralen- 


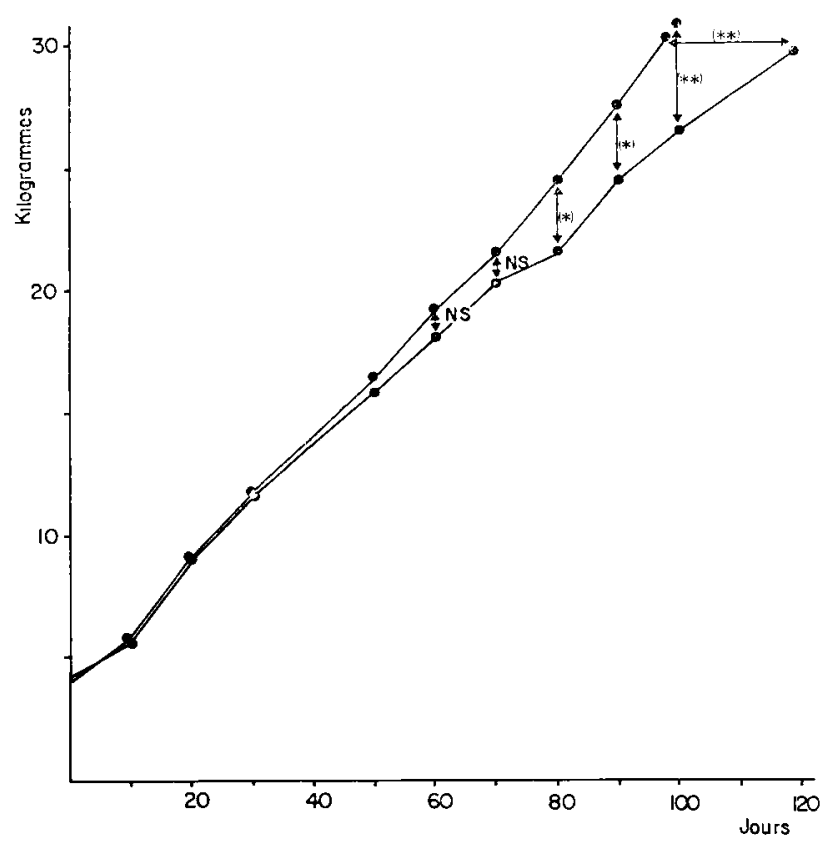

FIG. 2. - Variation du poids vif en fonction de l'áge et du sexe Seuil de signification : NS Effet non significatif

* Effet significatif $(\mathrm{P}<0,05)$

** Effet hautement significatif $(P<0,0 r)$

\section{TABLEAU I}

Variation de l'âge moyen à l'abattage en jours selon le sexe et le poids



Seuil de signification

NS Jiffet non significatif

+ Iffet significatif $(\mathrm{P} \leqslant 0.10)$

* Fffet significatif $(P \leqslant 0.05)$

** Jiffet hautement significatif $(P \leqslant 0.01)$ 
tissement de la croissance, s'accompagne aussi d'un dépôt de tissus adipeux plus important pour un même poids vif, et d'une consommation plus élevée en énergie pour obtenir le même poids des carcasses. De même, dans notre expérience, les femelles

TABLEAU 2

Quantité d'aliment concentré consommé en kg par les différents lots d'abattage

\begin{tabular}{c|c|c|c}
\hline \hline Sexe & \multicolumn{3}{|c}{ Poids abattage } \\
\cline { 2 - 3 } & $22 \mathrm{~kg}$ & $26 \mathrm{~kg}$ & $30 \mathrm{~kg}$. \\
Mâle & 14,6 & 21,5 & 28,9 \\
Femelle & 16,5 & 27,5 & 41,5 \\
\hline
\end{tabular}

ont consommé une quantité d'aliment pltus importante que les mâles (tab1.2) pour atteindre le même poids de carcasse. Les résultats rapportés dans le paragraphe suivant montrent aussi un plus grand développement des tissus adipeux des carcasses femelles, à poids égal.

\section{2. - Évolution des organes et des dépôts adipeux}

Le poids des organes étudiés (tab1. 3) augmente avec le poids à l'abattage. Il est également plus élevé chez les mâles que chez les femelles, à l'exception du gros intestin et du mésentère. Les réservoirs gastriques, l'intestin grêle et le gros intestin présentent une augmentation de poids linéaire en fonction du poids à l'abattage ; néanmoins, l'augmentation du poids de l'intestin grêle entre 22 et $30 \mathrm{~kg}$ de poids vif est faible. Ceci semble indiquer que cet organe s'est développé, avant $22 \mathrm{~kg}$ de poids vif, plus rapidement que les autres organes. Le sexe a eu une grande influence sur le développement de 1 'ensemble du système digestif $(\mathrm{P}<\mathrm{O}, \mathrm{OI})$.

Les dépôts adipeux des femelles (tabl. 4) ont été supérieurs à ceux des mâles, à partir de $22 \mathrm{~kg}$ de poids vif $(\mathrm{P}<0, \mathrm{OI})$. Les dépôts adipeux augmentent avec le poids à l'abattage, particulièrement chez les femelles à partir de $26 \mathrm{~kg}(805 \mathrm{~g}$ de graisse mésentérique et 7 I $9 \mathrm{~g}$ de graisse périrénale et pelvienne, à $30 \mathrm{~kg}$ de poids vif). Tandis que chez les mâles, ces dépôts adipeux restent presque inchangés entre 26 et $30 \mathrm{~kg}$.

Le poids de l'os du canon permet d'estimer l'importance du tissu osseux de la carcasse (Boccard, Dumont et PEYron, I958). Dans notre expérience, le poids du canon a augmenté avec le poids à l'abattage $(33,6 \mathrm{~g}, 34,4 \mathrm{~g}, 37,4 \mathrm{~g}$ à 22,26 et $30 \mathrm{~kg})$ et il est plus important chez les mâles que chez les femelles $(36,4$ et $33,8 \mathrm{~g})$, ce qui indique que dans les limites des poids étudiés, le squelette continue à se développer.

Une part importante du gain de poids entre 26 et $30 \mathrm{~kg}$ est donc constituée chez les femelles par un développement du tissu adipeux alors que chez les mâles, les tissus musculaires et osseux sont les principaux concernés. Ces observations sont en parfait accord avec les résultats des travaux de FourIE, KIRTON et JURY (I970) qui 


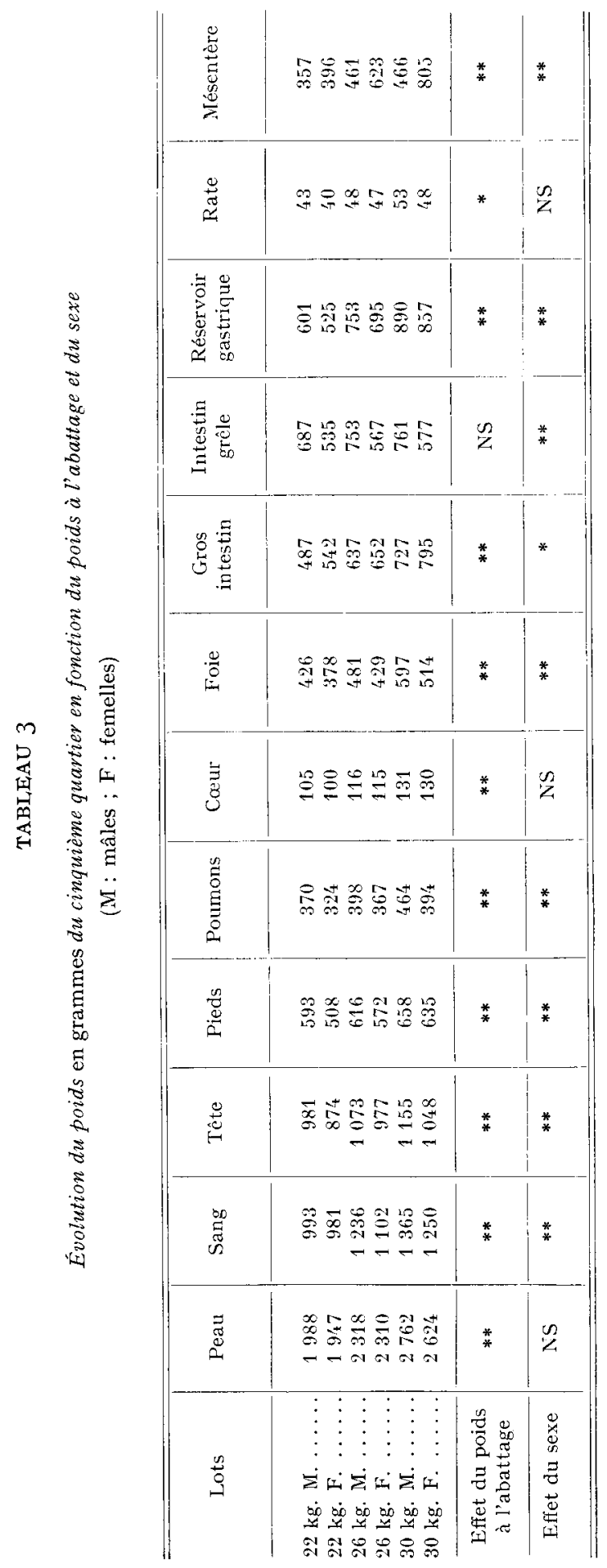


calculent des coefficients d'allométrie significativement différents entre sexes pour chacun des 3 tissus, adipeux, musculaire et osseux, les coefficients du tissu adipeux étant supérieurs pour les agneaux femelles et ceux des tissus musculaire et osseux plus élevés pour les mâles.

\section{TABIEAU 4}

Variation de l'importance du tissu adipeux en fonction du poids à l'abattage et du sexe (M : mâles ; F : femelles)

\begin{tabular}{|c|c|c|c|c|}
\hline Lots & $\begin{array}{c}\text { Graisse mésentérique } \\
(\mathrm{g})\end{array}$ & $\begin{array}{l}\text { Graisse périrénale } \\
\text { et pelvienne (s) }\end{array}$ & $\begin{array}{l}\text { Epaisseur C (I) } \\
(\mathrm{mm})\end{array}$ & $\begin{array}{c}\text { Epaisseur } C^{\prime}(\mathbf{1}) \\
(\mathrm{mm})\end{array}$ \\
\hline $22 \mathrm{~kg} . \mathrm{MI} . \ldots \ldots \ldots$ & 357 & 311 & 3.0 & 5.2 \\
\hline $22 \mathrm{~kg} . \mathrm{l}: \ldots \ldots \ldots$ & 596 & 635 & 4.4 & 6.2 \\
\hline $26 \mathrm{~kg} . \mathrm{M} . \ldots \ldots \ldots$ & 461 & 334 & 3.9 & 6.3 \\
\hline $26 \mathrm{~kg} . \mathrm{K} \ldots \ldots \ldots$ & $6 \div 3$ & 525 & 4.2 & $6 . t^{\prime}$ \\
\hline $30 \mathrm{~kg}$. .I. . . . . & 466 & 357 & 3.5 & 5.3 \\
\hline $30 \mathrm{~kg} . \mathrm{F} \ldots \ldots \ldots \ldots$ & 805 & 719 & 5.5 & 8.1 \\
\hline $\begin{array}{l}\text { Effet du poids } \\
\text { al l'abattage }\end{array}$ & $* *$ & * & NS & $\lambda S$ \\
\hline liffet du sexe & $* *$ & $* *$ & * & $*$ \\
\hline
\end{tabular}

(1) C : Épaisseur de la graisse de couverture a niveau de la première vertèbre lombaire. $C^{\prime}$ : Épaisseur de la graisse de couverture au niveau de la première vertèbre sacrée.

\section{3. - Caractéristiques des carcasses}

a) Rendements.

Le rendement commercial a été de 48,64 p. Ioo chez les mâles et de 5I,08 p. Ioo chez les femelles, les rendements vrais étant respectivement de $59,50 \mathrm{p}$. Ioo et $6 \mathrm{I}, 20$ p. IOO $(\mathrm{P}<0,0 \mathrm{I}$; tabl. 5). Les différences dans le contenu du tractus digestif, dans l'importance du cinquième quartier et dans les pertes par réfrigération des carcasses (KIRTON et BARTON, I962) expliquent les variations de rendement entre sexes.

En effet, la figure 3 montre bien la masse respective des composants principaux du poids vif des agneaux mâles et femelles abattus. Ainsi, les pertes par transport et jeûne sont de $6,04 \mathrm{p}$. Ioo chez les femelles et de 5,04 p. Ioo seulement chez les mâles. Cette différence, ajoutée à celle qui existait au départ de la bergerie (fréquence hebdomadaire des abattages et croissance plus élevée des mâles), fait que le poids des mâles est plus élevé avant l'abattage $(\mathrm{P}<0,05)$ Mais le contenu du tube digestif étant plus important chez les mâles que chez les femelles, le poids vif vide est identique pour les 2 sexes.

Les femelles possèdent un cinquième quartier moins développé que chez les mâles et en conséquence, leur carcasse chaude est plus lourde. Comme les pertes par réfrigération de ces carcasses sont inférieures à cause de leur plus grand état d'engrais- 
F. COLOMER-ROCHER, M. ESPEJO-DIAZ

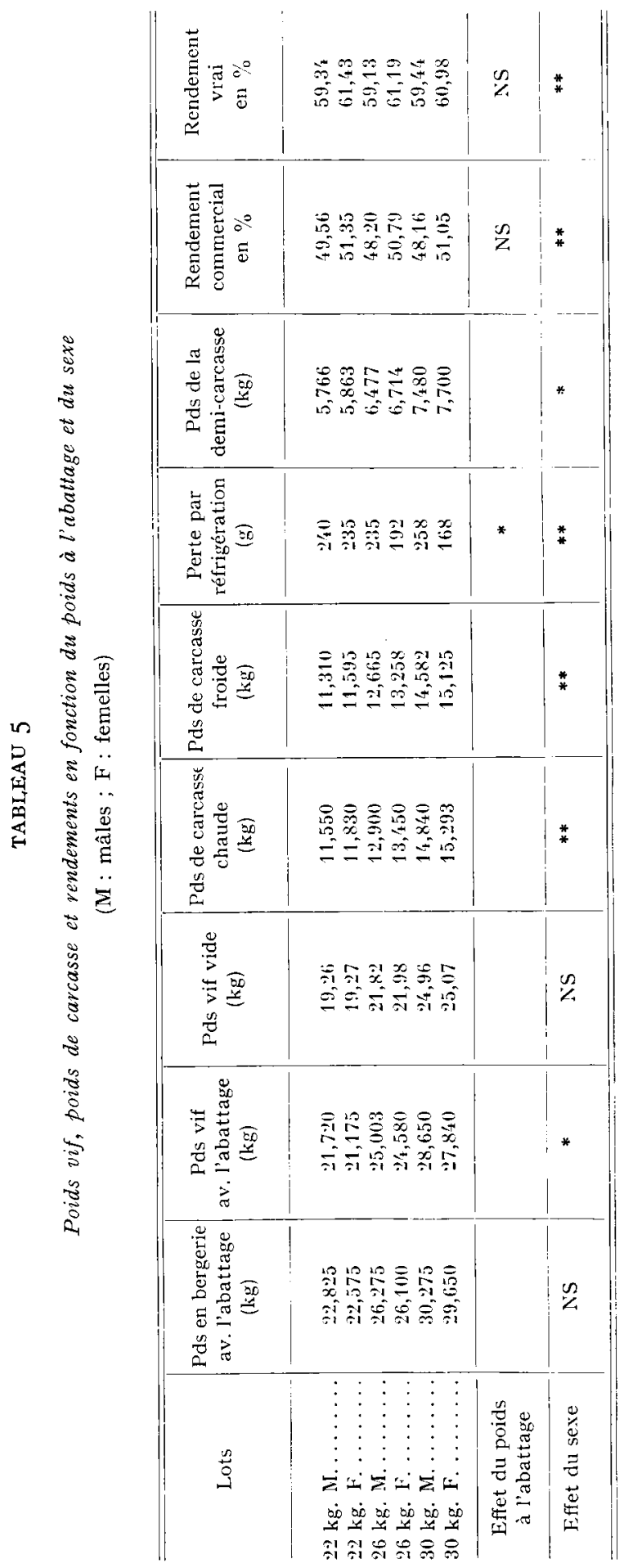


sement, les différences de poids observées entre les carcasses froides sont encore plus importantes, ce qui explique le rendement plus élevé des femelles.

Aucune variation du rendement n'est observée en liaison avec le poids d'abattage, l'augmentation du cinquième quartier ayant été proportionnelle à l'accroissement du poids des carcasses.

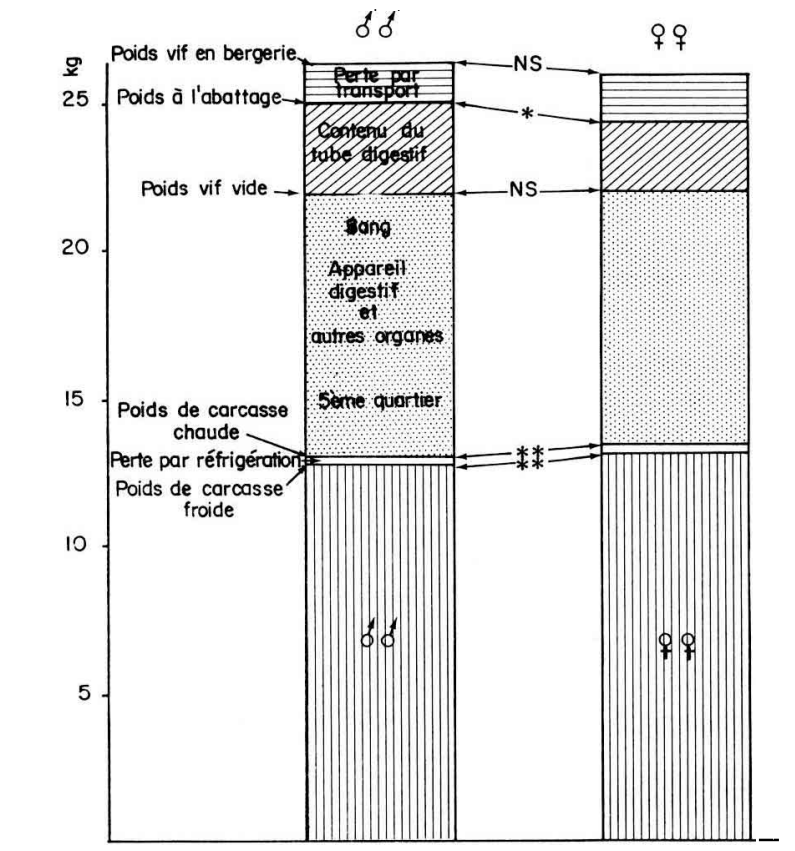

FIG. 3. - Poids vif des animaux et de leur carcasse selon le sexe

Seuil de signification : NS Effet non significatif

* Effet significatif $(\mathrm{P}<0,05)$

** Effet hautement significatif $(\mathrm{P}<\mathrm{o,OI})$

\section{b) Mesures sur les carcasses.}

Toutes les mesures obtenues, à l'exception de $\mathrm{K}$, ont augmenté avec le poids à 1'abattage (tabl. 6), l'augmentation longitudinale et volumique étant essentiellement fonction de l'accroissement de poids (BRODY, I945).

La mesure $\mathrm{F}$ croit peu par rapport aux autres mesures : l'augmentation en longueur des os du gigot est compensée surtout par des dépôts graisseux et aussi par l'épaississement des plans musculaires dans la région périnéale (BOCCARD et al., I96I ; Boccard, Dumont et Peyron, I964).

La conformation des carcasses peut être chiffrée à l'aide des relations proposées par ClARKE et Mc MEEKAN (I952) : une carcasse bien conformée doit être compacte et courte. Or, le tableau 6 montre que 1'indice de compacité du gigot $(\mathrm{G} / \mathrm{F})$, l'indice de rondeur de la poitrine (Wr/Th) et la relation profondeur/longueur ( $\mathrm{Th} / \mathrm{K}$ ) augmentent avec le poids de la carcasse, tandis que la relation longueur/largeur ( $\mathrm{K} / \mathrm{G})$ et profondeur/largeur $(\mathrm{Th} / \mathrm{H})$ diminuent. Ceci signifie donc qu'avec l'augmentation de poids les carcasses deviennent mieux conformées. On admet généralement qu'il 
existe une relation entre la conformation et l'état d'engraissement des carcasses. Cependant, on n'observe pas ici de différences, pour des carcasses de même poids, entre les mâles et les femelles pourtant plus grasses.

\section{TABLEAU 6}

Variation des mesures de la carcasse en fonction du poids à l'abattage

\begin{tabular}{|c|c|c|c|c|c|}
\hline \multirow[b]{2}{*}{$\begin{array}{l}\text { Mesures } \\
\text { de longueur } \\
\text { (mm) }\end{array}$} & \multicolumn{3}{|c|}{ Poids à l'abattage $(\mathrm{kg})$} & \multirow[b]{2}{*}{$\begin{array}{c}\text { Différence } \\
30-22\end{array}$} & \multirow[b]{2}{*}{\begin{tabular}{|c} 
Signification \\
des \\
différences
\end{tabular}} \\
\hline & 22 & 26 & 30 & & \\
\hline $\mathrm{F}$ & 271 & 268 & 277 & +6 & * \\
\hline G & 181 & 189 & 198 & +17 & ** \\
\hline $\mathrm{K}$ & 469 & 462 & 469 & 0 & NS \\
\hline $\mathrm{Wr}$ & 168 & 180 & 192 & +24 & ** \\
\hline Th & 227 & 233 & 237 & +10 & $* *$ \\
\hline \multicolumn{6}{|l|}{ Rapport } \\
\hline Wr/Th & 0,738 & 0,773 & 0,810 & & ** \\
\hline $\mathrm{G} / \mathrm{F}$ & 0,669 & 0,707 & 0,716 & & ** \\
\hline $\mathrm{K} / \mathrm{G}$ & 2,588 & 2,442 & 2,372 & & ** \\
\hline $\mathrm{Th} / \mathrm{G}$ & 1,256 & 1,233 & 1,199 & & * \\
\hline $\mathrm{Th} / \mathrm{K}$ & 0,486 & 0,506 & 0,506 & & * \\
\hline
\end{tabular}

Note : Le sexe n'a pas eu d'effet sur les mesures des carcasses.

c) Morceaux de découpe.

Le tableau 7 montre les effets du sexe et du poids à l'abattage sur les morceaux de la moitié gauche de la carcasse, d'une part en valeur absolue, d'autre part en valeur relative (pourcentage du poids de carcasse froide).

Il est intéressant de signaler que le pourcentage des morceaux de $\mathrm{I}^{\text {re }}$ catégorie (" pierna + costillar" = gigot + côtes + filet) n'a pas changé avec le poids à l'abattage ; par contre, le pourcentage de " espalda " (épaule) diminue et le pourcentage des "bajos" (poitrine) augmente en accord avec les résultats de BOcCARD, Dumont et LEFEVRe (I962) et de Fourie, KirTon et Jury (I970). Le pourcentage de "pierna " (gigot) a diminué légèrement comme le constatent également BoccARD, DUMONT et LEFÈVRE (I962) et KIRTON (I967).

L'influence $d u$ sexe se traduit par un pourcentage de morceaux de découpe de première catégorie plus élevé chez les femelles que chez les mâles $(\mathrm{P}<0, \mathrm{OI})$, supériorité essentiellement due à une plus grande proportion de "costillar " (côtes + filet). Les mâles présentent, par contre, un pourcentage plus élevé de " pierna " (gigot), " espalda " (épaule) et " cuello " (collier). Pour ce dernier morceau, on peut voir un début d'influence de caractère sexuel secondaire (BOCCARD, DUMONT et LEFÈVRE, Ig62). 


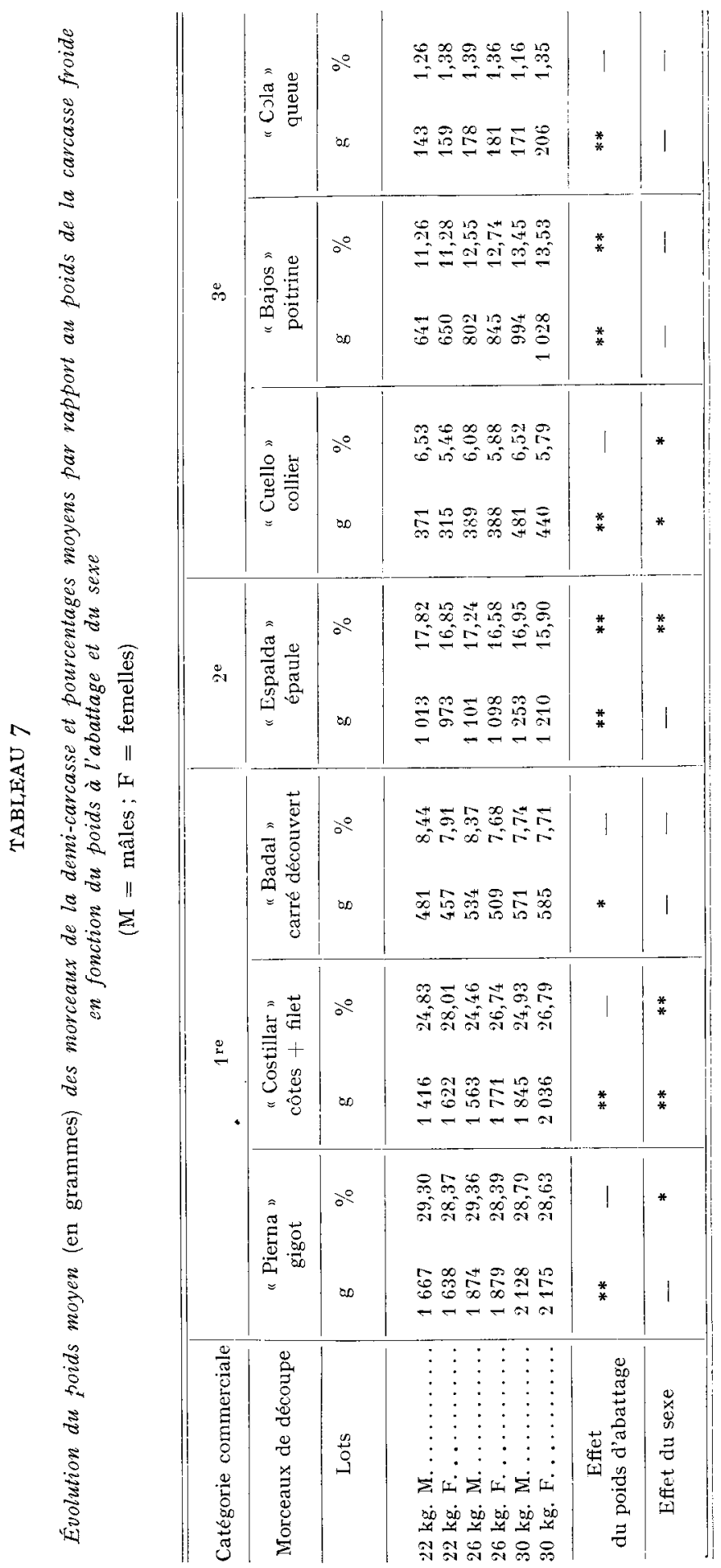




\section{IV. - CONCLUSIONS}

I. Le sexe des agneaux a une grande influence sur l'état d'engraissement des carcasses, mais aussi, plus généralement, sur leur précocité de développement : pour un poids donné, les femelles sont plus proches de leur développement adulte que les mâles. On peut observer des manifestations de ce phénomène dans la plus faible évolution du système digestif et du tissu osseux, et dans l'accroissement plus rapide de l'importance des dépôts adipeux internes et dorsaux pour les femelles que pour les mâles entre 26 et $30 \mathrm{~kg}$ de poids vif.

2. Cette différence de développement a des conséquences bouchères notables pour des animaux abattus au même poids. Les femelles ont un rendement plus élevé et une plus grande importance des morceaux de découpe dorsaux et ventraux (" costillar " et "bajos )), tandis que les mâles ont une plus grande proportion de "pierna » et " espalda " (gigot et épaule) ainsi que de morceaux présentant une base osseuse plus importante (" cuello " et " badal " = cou et carré découvert). On peut penser, en conséquence, qu'il serait intéressant de retenir le sexe comme critère additionnel pour la classification des carcasse d'agneaux.

On constate, par ailleurs, que l'augmentation du poids à l'abattage de 22 à $30 \mathrm{~kg}$, quel que soit le sexe, améliore la conformation des carcasses, sans que les rendements ni le pourcentage des morceaux de $I^{\text {re }}$ catégorie varient sensiblement.

3. I'appréciation bouchère des carcasses selon le poids et le sexe indique que dans les conditions d'alimentation de cet essai, les femelles issues du croisement industriel Manchego $\times$ Rasa Aragonesa devraient être abattues à $26 \mathrm{~kg}$ de poids vif. A un poids supérieur, les carcasses femelles présentent des dépôts adipeux excessifs. Par contre, les mâles abattus à $30 \mathrm{~kg}$ de poids vif n'ont pas présenté ces inconvénients, ce qui suggère qu'ils auraient pu être abattus à un poids vif encore plus élevé sans préjudice pour la qualité des carcasses.

4. La précocité de développement plus importante des femelles accroît également la différence de leur vitesse de croissance moyenne avec les mâles, particulièrement après le sevrage, de sorte que l'efficacité nutritionnelle des aliments distribués est d'autant plus faible par rapport aux mâles que l'on cherche à obtenir des carcasses plus lourdes.

La considération des deux facteurs mis ici en évidence (qualité des carcasses et consommation d'aliments) permet de conclure que l'on ne doit pas attendre des agneaux femelles, issus d'un croisement industriel, une aussi bonne valorisation commerciale que des mâles issus de ce même croisement.

Reçu pour publication en mars 1972.

\section{REMERCIEMENTS}

Nous tenons à remercier M. Cruañes Oliver, qui a mis les animaux de son troupeau à notre disposition, et la direction et le personnel de la Société kurstz (Matadero Industrial Frigorifico) qui ont permis la réalisation de la découpe et des mesures sur les carcasses. Nous sommes particulièrement reconnaissants à MM. BOcCARD, DUMONT et FLAMANT des conseils qu'ils nous ont prodigués au cours de la réalisation de l'expérience et lors de la rédaction de ce mémoire. 


\section{SUMMARY}

\section{INFLUENCE OF SLAUGHTER WEIGHT AND SEX ON CARCASS QUALITY OF CROSSBRED MANCHEGO $\times$ RASA ARAGONESA LAMBS}

Sixty Manchego $\times$ Rasa Aragonesa crossbred lambs were divided into six groups of ten animals each, $i$. e. 3 groups of each sex according to the slaughter weights $(22 \mathrm{~kg}, 26 \mathrm{~kg}, 30 \mathrm{~kg})$. All lambs were reared in identical conditions. The growth of the lambs, the yields, the fatness of the carcasses, the conformation and proportion of various cuts, were studied.

The males showed constant growth up to $30 \mathrm{~kg}$. The growth of the females was almost similar to that of the males during the first month, and afterwards it occurred at a reduced rate.

The growth rate reduction in the females was particularly noticeable after $22 \mathrm{~kg}$ live weight and after 80 days of age (fig. 2), depending on their greater physiological precocity. This decrease in growth rate was accompanied by higher fat deposits at given weights (table 4 ) and by greater feed intakes (table 2 ) to obtain the same carcass weights.

The commercial and true yields of the males were lower than those of the females $\left(4^{8.64}\right.$ p. 100 and $59.60 \mathrm{p}$. Ioo versus $5 \mathrm{I.08}$ p. Ioo and 6r.20 p. Ioo) which can be attributed to the following factors : larger digestive organs, higher weight of the fifth quarter, higher losses from refrigeration and less fat (fig. 3). The conformation of the carcasses improved with the slaughter weight, but in the females the increase was accompanied by excessive fat. The percentage of first category cuts of the female carcasses was higher (63.4 I p. I00) than that of the male carcasses $(62,07$ p. roo). This can be clue to a greater degree of fatness. In general, a higher precocity in the development was noted in the females. It may be concluded that for the crossbred animals studied and under the conditions of rearing and feeding described, the optimum slaughter weight of the females was $26 \mathrm{~kg}$ because of excessive deposit of fat in heavier carcasses. The males may be slaughtered at live weights exceeding $30 \mathrm{~kg}$.

\section{RÉFÉRENCES BIBLIOGRAPHIQUES}

Boccard R., Dumont B.-L., PEYron C., I958. Valeur de quelques mensurations pour apprécier la qualité des carcasses d'agnealix. 4th Meet. Europ. Meat. Research Workers. Cambridge, sept., I.5, 9.

Boccard R., Deront B.-L., Le Guelte P., Arnoux J., ig6i. Étude de la production de la viande chez les ovins. IV. Relation entre la forme et la composition du membre postérieur. Ann. Zootech., 10, I55-I 60 .

Boccard R., Duplan J.-M., ig6r. Etude de la production de la viande chez les ovins. III. Note sur l'influence de la vitesse de croissance sur la composition corporelle des agneaux. Ann. Zootech, 10, 3 I-38.

Boccard R., Duront B.-L., Lefère J., 1962, Étude de la production de la viande chez les ovins. V. Note sur la croissance relative des régions corporelles de l'agneau Ann. Zootech., 11, $257-262$.

Boccard R., Dumont B.-L., Peyron C., I964. Étude de la production de viande chez les ovins. VIII. Relations entre les dimensions de la carcasse d'agneaux. Ann. Zootech., 13, 367-378.

Brody S., I945. Bio-energetics and growth. Reinhold Publ. Corp., New York.

Clarke J. A., Mc Meekan C. P., I952. New Zealand Lamb and Mutton. N. Z. J. Sci., Techn., A. 33, I-I5.

Desvignes D., Cattin-Vidal P., Poly J., ig66. Comparaison de la valeur de divers types de croisement industriel pour la production d'agneaux de boucherie. I. Croissance pondérale des agneaux. Ann. Zootech., 15, 47-66.

Flamant J.-C., Boccard R., ig66. Estimation de la qualité de la carcasse des agneaux de boucherie. Ann. Zootech., 15, 89-II3.

Flamant J.-C., Cattin-Vidal P., Poly J., ig67. Comparaison de la valeur de divers types de croisement industriel pour la production d'agneaux de boucherie. II. Valeur bouchère des agneaux. Ann. Zootech., $16,4 \mathrm{I}-63$.

Fourie P. D., Kirton A. H., Jury J. E., I970. Growth and development of sheep. II. Effect of breed and sex on the growth and carcass composition of the Southdown and Romney and their cross. N.Z.J. Agric. Rev., 13, 753-770.

Kemp J. D., Crouse J. D., Deweese W., Moody W. G., r97o. Effect of slaughter weight and castration on carcass characteristics of lambs. J. Anim. Sci., 30, 348-354. 
KIrTon A. H., Barton R. A., I962. Studies of some indices of the chemical composition of lamb carcasses. J. Anim. Sci, 21, 553-557.

Kirton A. H., I967. Conformation and lamb carcass quality. Sheep Farming Annual, 196\%, ir3-I26. Lambuth T. R., Kemp J. D., GLimp H. A., I970. liffect of rate of grain and slaughter weight on lamb carcass composition. J. Anim. Sci., 3J, 27-35.

Lucas A. I. M., r97I. (Communication personnelle).

Palsson H., 1939. Meat qualities in tho sheep : special reference to scottish breeds and crosses. $J$. Agric. Sci., 23, 544.

Palsson H., Vfrges J. B., 1952. Effects of the plane of nutrition on growth and the clevelopment of carcass quality in lambs. Part $\mathbf{I}_{-2}$. The effects of high and low planes of nutrition at different ages. $J$. Agric. Sci., 42, I-I 49 .

Sanchez-Belda A., I97o. Estudio crítico de la prodıcción y comercialización de la carne en España. Ganada ovino. Tribuna Veterinaria, no $\mathbf{3}, \mathbf{4}$ y $\mathbf{5}$. 